\title{
Mastoid Dressing in Ear Surgery: Is it Still Relevant?
}

https://doi.org/10.47210/bjohns.2021.v29i2.477

Rahul Naga, ${ }^{1}$ Tejpal Singh Bedi, ${ }^{1}$ Renu Rajguru, ${ }^{1}$ Inderdeep Singh, ${ }^{1}$ Saurabh Mahajan ${ }^{1}$
$\underline{\text { Introduction }}$
Mastoid dressings are conventionally used in patients who undergo Tympanoplasty using post auricular approach. The rationale behind using a mastoid dressing is that the said dressing prevents haematoma formation and acts as a protective covering during the post-operative period. This study aims to understand the significance of mastoid dressing in patients undergoing Cortical Mastoidectomy and Tympanoplasty and its role in preventing post-operative wound complications and patient comfort.
Materials and Methods
A total of 77 patients were enrolled in the study over a one year period and were randomised into dressing and no dressing groups with the aim of deciphering any advantage of the mastoid dressing over a smaller dressing.
$\underline{\text { Results }}$
Our data revealed no added advantage of conventional mastoid dressing over a small gauze piece dressing in terms of haematoma/seroma formation, contusion and wound dehiscence. However, there was significantly reduced patient discomfort and sleeping difficulties post-operatively in the no dressing group.
Conclusion
Smaller dressing is more favourable than the bulkier conventional mastoid dressings.
Keywords
Tympanoplasty; Mastoidectomy; Bandages; Seroma; Hematoma

ABSTRACT

$\mathrm{M}$ astoid dressings are conventionally used in patients who have undergone Tympanoplasty using a post auricular approach. The rationale behind using a mastoid dressing remains the belief that the said dressing prevents hematoma formation and acts as a protective covering during the post-operative period. However when Hill et al. studied the average pressure exerted by a mastoid dressing using a balloon catheter and pressure transducer, they concluded that a mastoid dressing lost the required pressure to prevent a hematoma post 1 hour of its use. ${ }^{1}$ Hence, there was little scientific backing towards its use in preventing

1 - Department of ENT, INHS Asvini, Colaba Mumbai

2 - Department of PSM, AFMC, Pune

\section{Corresponding author:}

Dr Tejpal Singh Bedi

email: tsbedi26@gmail.com hematoma. The dogma surrounding this conventional dressing also remains to be questioned in terms of patient comfort. Current advances in haemostatic techniques usually ensure a dry post-operative field and thus the very practice seems to need a scientific reconsideration. Khan et al in their systematic review of mastoid dressing also conclude that no advantage is offered in uncomplicated, routine middle ear surgeries. ${ }^{2}$ Lou et al. after their work on mastoid dressing post Cochlear Implant (CI), have also favoured abandoning the use of mastoid bandage post uncomplicated CI. ${ }^{3}$

O'Brien et al have curtailed the time of mastoid dressing to 2 hours post-surgery and recommended that the patients be discharged post removal; and that the presence of a head dressing must not be a factor in decision making for a day care procedure or not. ${ }^{4}$ However, keeping in mind the Indian populace with a majority of patients belonging to a lower socio-economic strata. ${ }^{5}$ Prudence dictates that the said recommendations 


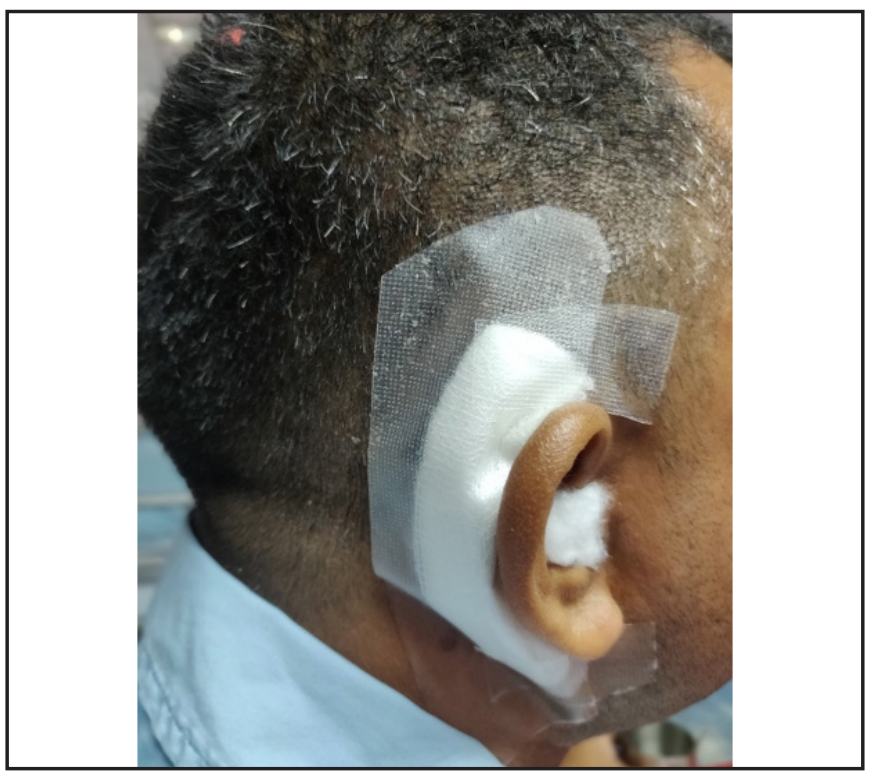

Fig. 1. A patient with single gauze piece dressing secured using adhesive tape.

can only be accepted in India after due deliberation.

This study aimed to understand the significance of mastoid dressing in patients undergoing Cortical Mastoidectomy and Tympanoplasty via the post-aural approach.

\section{Materials and Methods}

This prospective randomized double-blinded study was performed at a tertiary care centre. Non-smokers aged 18-60 years with no Auto-immune disorders / immunocompromised state / diabetes mellitus / anticoagulant use undergoing cortical mastoidectomy and tympanoplasty were chosen for the study. The study included all patients fitting into the above criteria from 01 May 2019- 01 May 2020.

The patients underwent Cortical Mastoidectomy and Tympanoplasty using a post-aural approach and standard surgical steps; the closure was done in layers and meticulous haemostasis, as is normal practice, was undertaken. Post-closure the patients were randomly assigned to receive either a single gauge piece cover stabilized with adhesive tape over the post aural incision (Fig. 1) or the conventional mastoid dressing (Fig. 2)

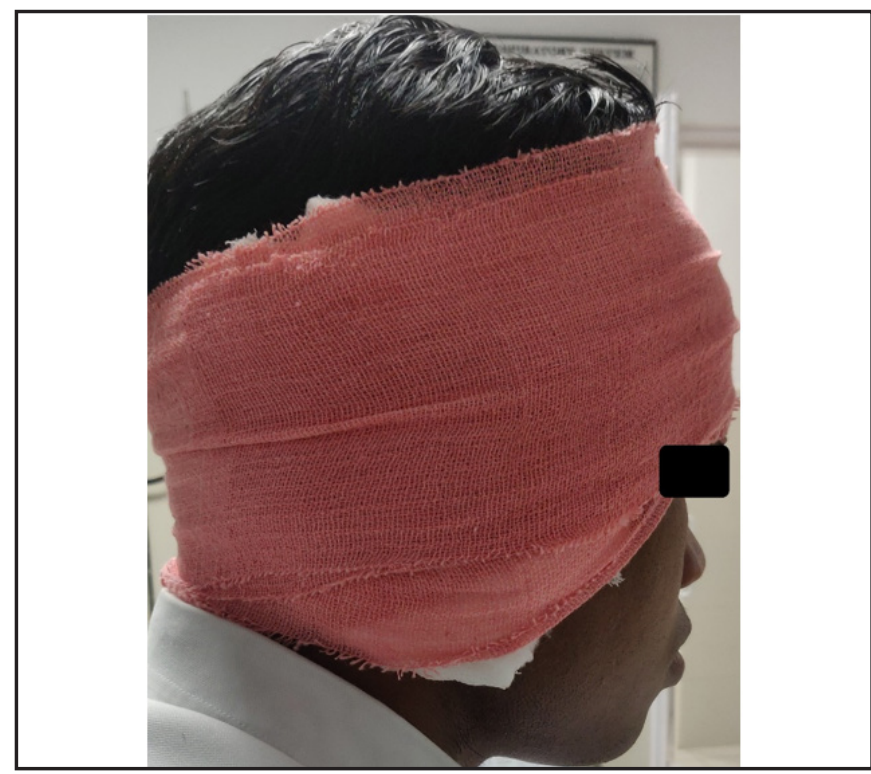

Fig. 2. A patient with conventional bulky mastoid dressing.

based on a lottery system. The surgeon and patient were blinded to the randomization and all surgeries were performed by a single surgeon to rule out bias. The mastoid dressing and simple dressing were removed on post-operative day 01 .

Patients were evaluated for any haematoma/seroma formation, contusion/erythema post removal and subsequently a week later for any evidence of wound dehiscence post-suture removal. In addition, patients were also quizzed about pain on dressing removal, neck pain and sleeping difficulty, if any, that could be attributed to the type of dressing used.

\section{Results}

A total of 77 patients were enrolled in the study over a period of one year of which 36 received a conventional mastoid dressing and 41 received a single gauze piece secured using an adhesive tape. The age of participants ranged from 19-60 with a mean of 36.32 and a standard deviation of 11.16. Out of the total patients 40 were male and 37 were female (Fig. 3).

Of the above lot we found that in the mastoid dressing group 2 patients developed a hematoma/ seroma; 7 developed contusions and 2 patients landed 


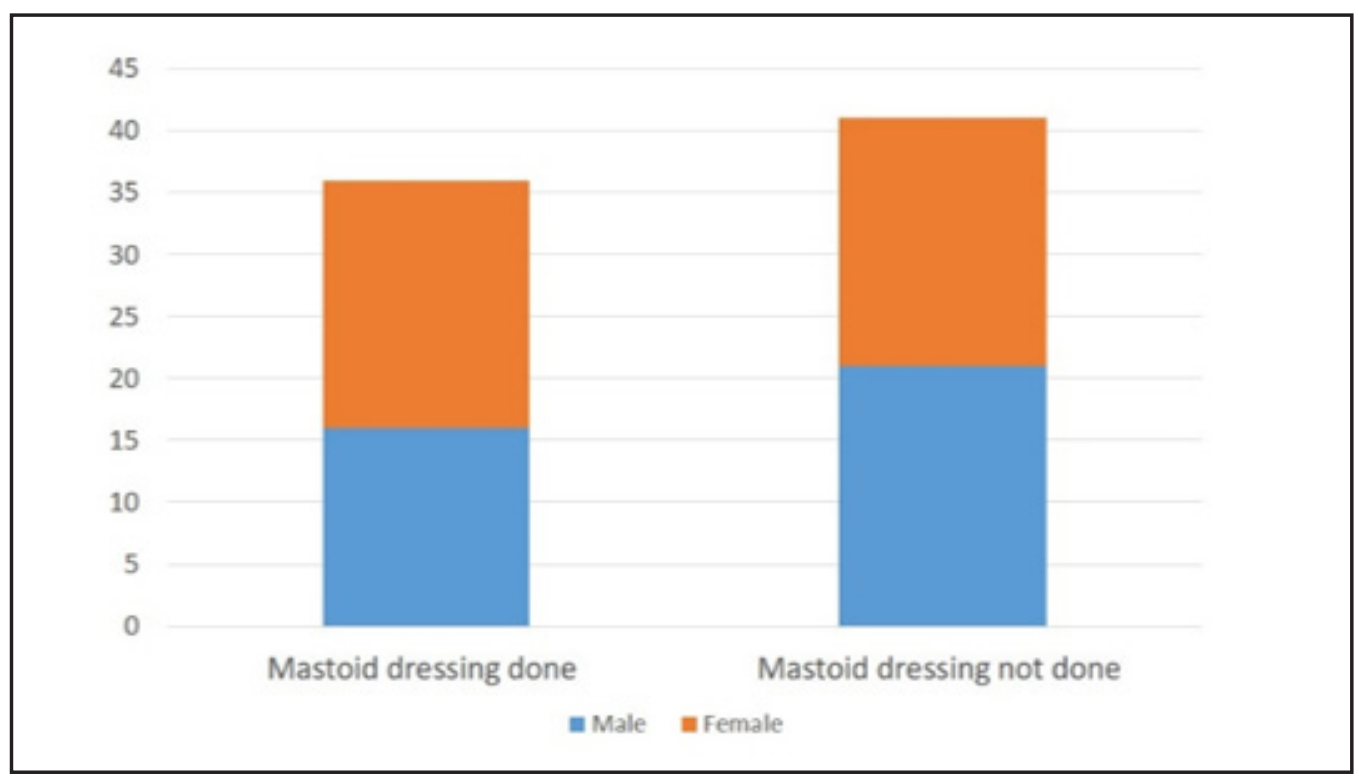

Fig. 3. The sex distribution of participants in both groups

up with wound dehiscence; 31 patients reported sleeping difficulty and 21 patients reported discomfort in the form of headache/neck pain due to the dressing. While in the no mastoid dressing group, 1 patient developed a hematoma/seroma and 3 patients had wound dehiscence, 2 patients reported contusion. Only 8 patients reported discomfort due to dressing because of painful removal and 3 patients reported sleeping difficulty.

Evaluation of the said data using Fisher's exact test revealed no statistical significance in the rates of hematoma/seroma formation, wound dehiscence or contusion with $\mathrm{p}$ values of $0.596,1$ and 0.074 respectively; the results were far more promising in view of sleeping difficulty and discomfort due to dressing with a $p$ value of $<0.001$ in both groups (Table I).

Thus, revealing that there was no added advantage of the dressing in terms of haematoma/seroma formation/ wound dehiscence and contusion; however, there was a significantly less sleeping difficulty and decreased pain in the no dressing group.

\section{Discussion}

The term Tympanoplasty was coined by Wullstein in 1953 and is commonly performed using a retro or postauricular approach as described by Wilde. ${ }^{6,7}$ The use of a mastoid dressing has been a convention practiced since temporalis fascia graft usage in the 1950's by Heerman. ${ }^{4,8}$ However, Hill et al proved that the actual pressure indices of a mastoid dressing are inadequate to prevent hematoma formation. ${ }^{1}$ Rowe-Jones et al brought the practice in question in 1993 with a series of 100 cases, while Castelli et al performed a study on over 400 individuals during a 6 year period..${ }^{9}{ }^{10}$ Both the works concluded that with uncomplicated middle ear surgeries, the use of a mastoid dressing can be done away with. Lou et al further questioned the use of a mastoid dressing in uncomplicated Cochlear Implant. ${ }^{3}$ The meta-analysis of mastoid dressings by Khan et al, again concluded in favour of abandoning the conventional mastoid dressing. ${ }^{2}$ Interestingly, Okur et al in their study concluded that a mastoid dressing has no influence on the ear-helix distance post surgery as well. ${ }^{11}$

It can be argued logically that with modern haemostatic techniques, the use of the cumbersome mastoid dressing has become redundant. Our study also tried to qualitatively adjudge the sleeping difficulties and discomfort associated with the dressings. A few cases in the no mastoid dressing group did claim that the removal was painful, on the other hand, a majority of cases with the conventional mastoid dressing experienced sleeping difficulty/headache and neck pain. The dressing per se, adds to no favourable outcome, 
Table I: The p-values of various parameters

\begin{tabular}{|c|c|c|c|c|}
\hline S. NO. & GROUP & $\begin{array}{c}\text { MASTOID } \\
\text { DRESSING DONE }\end{array}$ & $\begin{array}{c}\text { MASTOID } \\
\text { DRESING NOT } \\
\text { DONE }\end{array}$ & P-VALUE \\
\hline 1 & Haematoma/seroma & $2 / 36$ & $1 / 41$ & 0.596 \\
\hline 2 & Wound dehiscence & $2 / 36$ & $3 / 41$ & 1 \\
\hline 3 & Contusion & $7 / 29$ & $2 / 41$ & $<.074$ \\
\hline 4 & Sleep difficulty & $31 / 36$ & $8 / 36$ & $<0.001$ \\
\hline 5 & $\begin{array}{c}\text { Discomfort due to } \\
\text { dressing }\end{array}$ & $21 / 36$ & $8 / 41$ & $<0.001$ \\
\hline
\end{tabular}

as is evident from the lack of any statistical difference between the two groups when compared for haematoma/ seroma formation, contusion and wound dehiscence. In addition, the dressing is bulky and causes discomfort. While, a single gauze piece dressing is aesthetically more favourable and does not interfere with sleep and causes significantly less discomfort, as was evident from the analysed data. The patients thus, are more comfortable the next morning.

At the outset, there was some concern in our minds regarding frequent soakage of the gauze piece, which would require changing and hence frequent ward visits. However, the gauge pieces remained in situ with minimal soakage in almost all the cases. The cotton ball placed outside the EAC did require frequent changing, the same was conveyed to the patient and good compliance was experienced. The move from conventional mastoid dressing to smaller dressing would also stand favourably in large centres, as it will bring down the cost of the surgery.

Our data, largely reveals similar trends as compared to other studies done internationally. However, the other studies did not qualitatively assess for sleeping difficulties and pain/headache associated with the two groups.

Based on our review of literature and PubMed search, this is the only prospective randomized study carried out in the Indian context. Moreover, unlike none of the similar studies; we have selectively chosen patients who have undergone a cortical mastoidectomy and tympanoplasty for the purpose of the study.

It does appear that conventional mastoid dressing should be abandoned in favour of single gauge piece dressing, however our study is based on a relatively small sample size.

\section{Conclusion}

We conclude that conventional mastoid dressing be abandoned for the more favourable smaller dressing. The smaller dressing is equally efficacious and has the additional advantage of being more patient friendly in terms of aesthetics, sleeping comfort and cost.

\section{References}

1. Hill J, Allan W, Malhan D, Williams ED. Pressure exerted by head bandages used in otological surgery. J Laryngol Otol. 1993; 107:1110-2

2. Khan I, Mohamad S, Ansari S, Iyer A. Are head bandages really required after middle-ear surgery? A systematic review. J Laryngol Otol. 2015; 129(8):740-3. doi:10.1017/ S0022215115001565

3. Luo SD, Su CY, Wu CM, Hwang CF. Mastoid pressure dressing for cochlear implantation-is it necessary? Int J Pediatr Otorhinolaryngol. 2009; 73: 857-60

4. O'Brien JX, Ruskin O, Price T. Pressure dressings in mastoid and middle ear surgery: are they necessary? A retrospective review of patient outcomes. ANZ Journal of Surgery. 2018; 88: 1047-50

5. Agrawal A, Bhargava P. Comparative Evaluation of Tympanoplasty with or Without Mastoidectomy in Treatment 
of Chronic Suppurative Otitis Media Tubotympanic Type. Indian J Otolaryngol Head Neck Surg. 2017; 69(2):172-5

6. Aina J. Gulya, Lloyd B Minor, Dennis S Poe. GlasscockShambaugh Surgery of the Ear, 6th edn. USA:People's Medical Publishing House; 2003

7. Sarkar S. A review on the history of tympanoplasty. Indian J Otolaryngol Head Neck Surg. 2013; 65(Suppl 3):455-60

8. Parida PK, Nochikattil SK, Surianarayanan G, Saxena SK, Ganesan S. A comparative study of temporalis fascia graft and vein graft in myringoplasty. Indian J Otolaryngol Head Neck
Surg. 2013; 65(Suppl 3):569-74

9. Rowe-Jones JM, Leighton SE. The value of head dressings for middle ear surgery. J Laryngol Otol. 1993; 107:17-9

10. Castelli ML, Di Lisi D, Marcato P, Tavormina P, Cappellaro E, Sartoris A. Is pressure dressing necessary after ear surgery? Ann Otol Rhinol Laryngol. 2001;110:254-6

11. Okur E, Kahveci OK, Miman MC, Y1ldız H, et al. Ear protrusion after tympanoplasty and the use of mastoid dressing. Kulak Burun Bogaz Ihtis Derg. 2014; 24(2):74-7. doi: 10.5606/ kbbihtisas.2014.94758. PMID: 24835901. 